\title{
Efficient Re-degaussing Technique for a Naval Ship Undergoing a Breakdown in Degaussing Coils
}

\author{
Dong-Wook Kim ${ }^{3}$, Sang-Kyun Lee ${ }^{1}$, Byungsu Kang ${ }^{1}$, Jeonghun $\mathrm{Cho}^{4}$, WooSeok Lee ${ }^{2}$, \\ Chang-Seob Yang ${ }^{3}$, Hyun-Ju Chung ${ }^{3}$, and Dong-Hun Kim ${ }^{1 *}$ \\ ${ }^{1}$ Department of Electrical Engineering, Kyungpook National University, Daegu 41566, Korea \\ ${ }^{2}$ Department of Electric Automatization, Busan Institute of Science and Technology, Korea \\ ${ }^{3}$ The 6th R\&D Institute, Agency for Defense Development, Changwon 51678, Korea \\ ${ }^{4}$ School of Electronics Engineering, Kyungpook National University, Daegu 41566, Korea
}

(Received 5 February 2016, Received in final form 4 May 2016, Accepted 9 May 2016)

\begin{abstract}
A naval ship equipped with a degaussing system may undergo a breakdown in degaussing coils at sea. In the case, underwater magnetic field around the ship abruptly grows up and it can make the ship be easily exposed to fatal hazards such as magnetic mines or torpedoes. This paper proposes an efficient and practical redegaussing technique for a ship where a part of degaussing coils is out of order. To achieve this, an analytical design sensitivity formula and approximated degaussing coil field are exploited, and then new optimum currents of available coils are reassessed. To validate the proposed method, a muck-up ship equipped with 14 degaussing coils is tested in scale-down magnetic treatment facilities under three faulty coil conditions.
\end{abstract}

Keywords : degaussing, electromagnetics, inverse problem, design sensitivity

\section{Introduction}

In the earth's magnetic field, the ferromagnetic hull of a naval ship is magnetized and accordingly it induces underwater magnetic field around the ship. To mitigate such induced magnetic (IM) field, vessels are usually equipped with several tens of onboard degaussing coils consisting of longitudinal $(L)$, athwartship $(A)$ and vertical $(V)$ coils (refer to Fig. 1) [1-8]. The principle of the degaussing technique is to generate degaussing coil field of which the magnitude is same as that of IM field due to the hull, but two fields (i.e. IM field and coil field) have opposite directions with each other [4]. In order to minimize IM field, individual coil currents are elaborately tuned in specific magnetic treatment facilities (MTF), where measurement, control and monitoring systems for underwater magnetic field are installed [2, 3, 5-8]. After degaussing treatment, IM field can be reduced by nearly $90 \%$ of its original magnitude. However, it may happen that a breakdown in a part of degaussing coils occurs at sea. Then, the magnitude of underwater magnetic field

(C)The Korean Magnetics Society. All rights reserved.

*Corresponding author: Tel: +82-53-950-5603

Fax: +82-53-950-5603, e-mail: dh29kim@ee.knu.ac.kr abruptly grows up even though one of degaussing coils is out of order. Consequently, it is liable to expose the ship to fatal hazards such as magnetic mines or torpedoes. In such emergency situation, the best way is to reduce IM field as much as possible through utilizing available degaussing coils except for a faulty coil. It means that new optimum currents have to be allotted to available coils not in MTF but at sea. Herein, a technique for executing such emergency measure is called re-degaussing.

In this paper, an efficient and practical re-degaussing technique is proposed for a ship where a part of degaussing coils is out of order. Under the limited information of normal degaussing coil currents and coil arrangement, new optimum currents of available coils must be reestimated. To achieve this, the method exploits an analytical design sensitivity formula with respect to the magnetomotive forces (mmf) of degaussing coils and degaussing coil field approximated by a field integral equation. To validate the proposed method, a model ship equipped with 14 degaussing coils is tested in a scaledown MTF test room under three faulty coil scenarios, and predicted re-degaussing results are compared with experimental ones. 


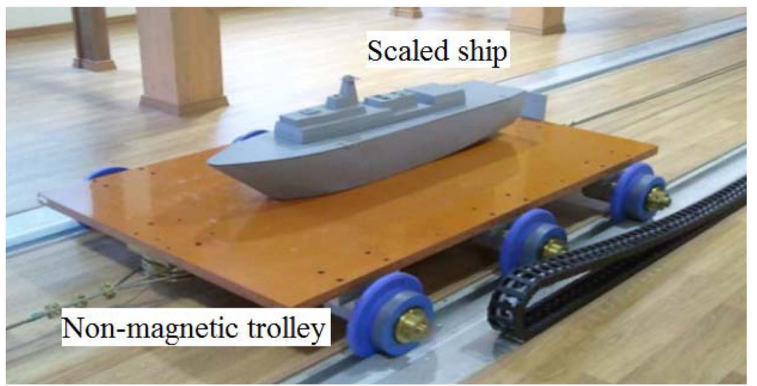

(a)

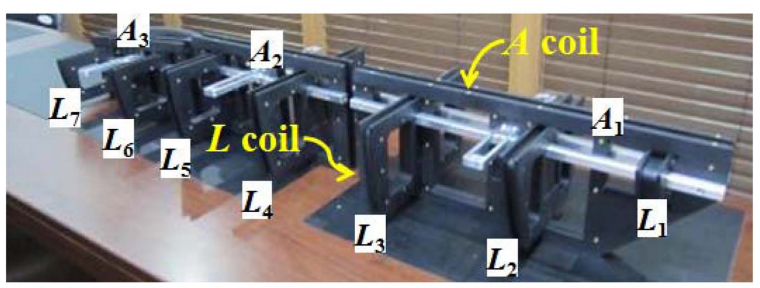

(b)

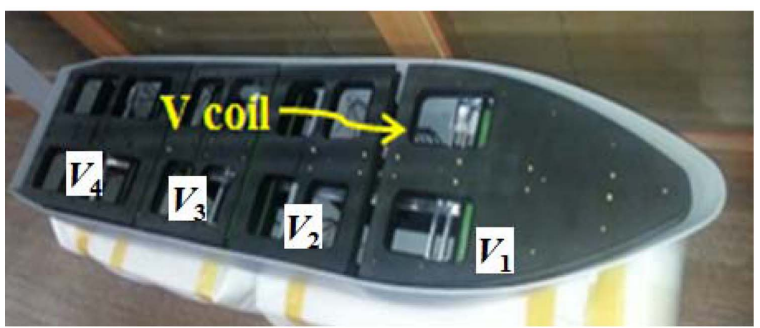

(c)

Fig. 1. (Color online) Photos of a model ship and degaussing coils: (a) Mockup, (b) $V$ and $A$ coil array, (c) $V$ coil array.

\section{Degaussing of a Scaled Ship}

For a better understanding of the goal of this paper, the degaussing technique along with a mockup ship and scaledown MTF systems is briefly explained. The setup of the MTF test room is described and accordingly the degaussing procedure is presented.

\subsection{Scale-down MTF test room}

The mockup was made of steel plate with a length of $1,514 \mathrm{~mm}$, width of $303 \mathrm{~mm}$ and height of $210 \mathrm{~mm}$. Degaussing coils consisting of four $V$ coils, seven $L$ coils, and three $A$ coils were installed in the ship as seen in Fig. 1 where a trolley and coil bobbins were made of nonmagnetic materials.

The MTF test room for scaled ships was built up of non-magnetic materials illustrated in Fig. 2 where power supply, control and monitoring units are not included. The large rectangular coils in Fig. 2 can simulate earth magnetic field at any place on the earth. The tri-axial magnetic sensors laid underground measure magnetic field signals around the ship moving in a north-south direction. The

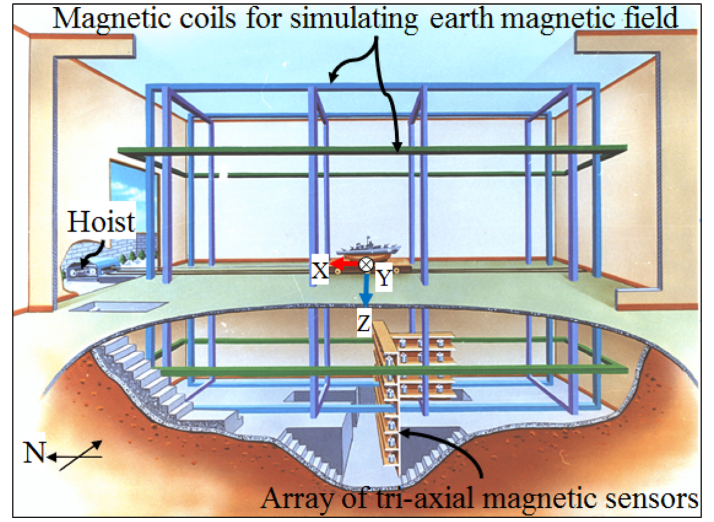

Fig. 2. (Color online) Schematic of a scale-down MTF test room.

degaussing coils are individually driven by direct current suppliers, and accordingly coil currents and underground magnetic field signals are recorded in real time.

In the MTF test room, three magnetic treatments consisting of field signal separation, deperming and degaussing are carried out for magnetic silencing of the ship. Apart from IM field, the hull itself possesses permanent magnetization due to mechanical/thermal stress during construction and operation. Actually, magnetic field created by the hull corresponds to the sum of IM and permanent magnetic (PM) fields before the magnetic treatments. To conduct effective deperming and degaussing, the two field components (i.e. IM and PM fields) have to be exactly identified through the field signal separation process. Then, the deperming process is executed to reduce the permanent magnetization on the hull as much as possible. The last treatment of degaussing is to minimize IM field as well as PM field remaining after the deperming.

\subsection{Degaussing procedure}

Through the field signal separation, IM field of the ship is easily separated from PM one. Before degaussing, three normalized components of IM field measured at a depth of $0.345 \mathrm{~m}$ under the keel line are shown in Fig. 3. Herein, the degaussing procedure for only IM field is explained because PM field is degaussed by the same way.

As mentioned early, the core technique of degaussing is to obtain optimum current values of individual degaussing coils of which the composite field waveforms coincide with those of IM field. In usual, the degaussing coil field due to the coil mmf includes a shielding effect of the hull. However, it is difficult in precisely predicting the shielding effect by numerical field analysis methods because an elaborate hull modeling is very complicated in three dimensions. To tackle the problem, reference coil field data and its field linearity relative to the coil $\mathrm{mmf}$ are exploited in 


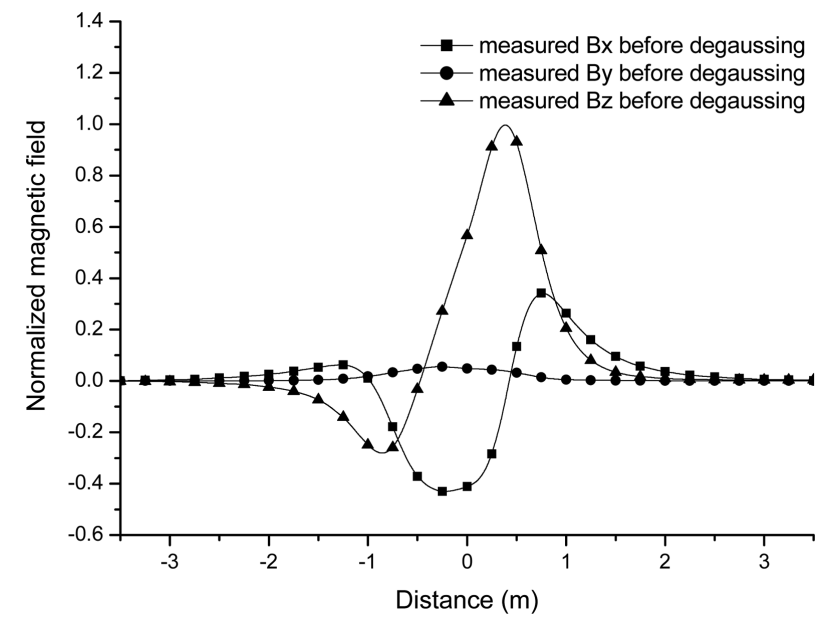

Fig. 3. Measured IM field components before degaussing.

real MTF instead of very cumbersome numerical field solutions [8].

The reference coil field including the shielding effect is measured as feeding a certain mmf value $\mathfrak{I}_{R}$ to an individual coil, one by one. Three directional components of the reference coil field $\mathrm{B}_{j}$ are illustrated in Fig. 4 where the $j$ th $\mathrm{V}$ coil field waveforms are displayed along with the $j$ th $\mathrm{L}$ coil ones. Since the coil field magnitude is the order of milli-gauss, the field linearity is guaranteed within a maximum degaussing current permitted. After all, the coil field $\mathrm{B}$ generated by any $\mathrm{mmf}$ value $\mathfrak{I}$ is easily estimated by Eq. (1).

$$
\mathbf{B}=\mathbf{B}_{j} \frac{\mathfrak{I}}{\mathfrak{I}_{R}} \quad j=1, \cdots n p
$$

where $n p$ is the number of observation points.

The last process is to highly tune individual coil currents so as to reduce IM field as much as possible. For the purpose of doing this, the optimization technique called a design sensitivity method in conjunction with the reference coil field data was adopted [7, 8]. Fig. 5(a) shows three

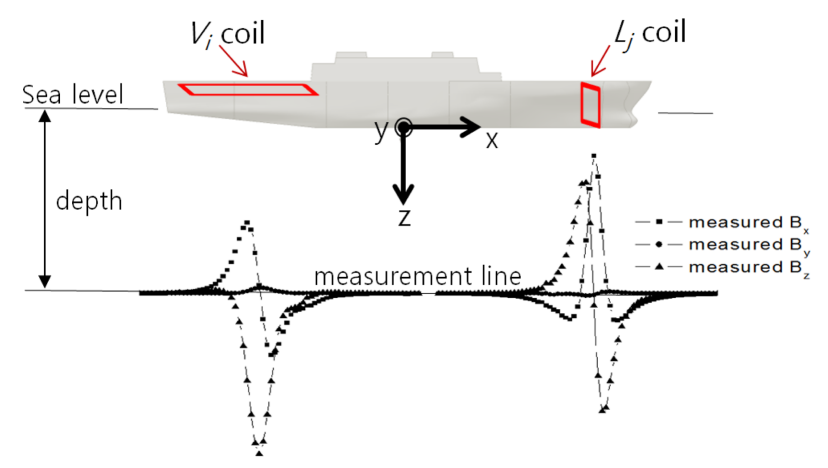

Fig. 4. (Color online) Reference coil field data measured for $V_{j}$ and $L_{j}$ coils.

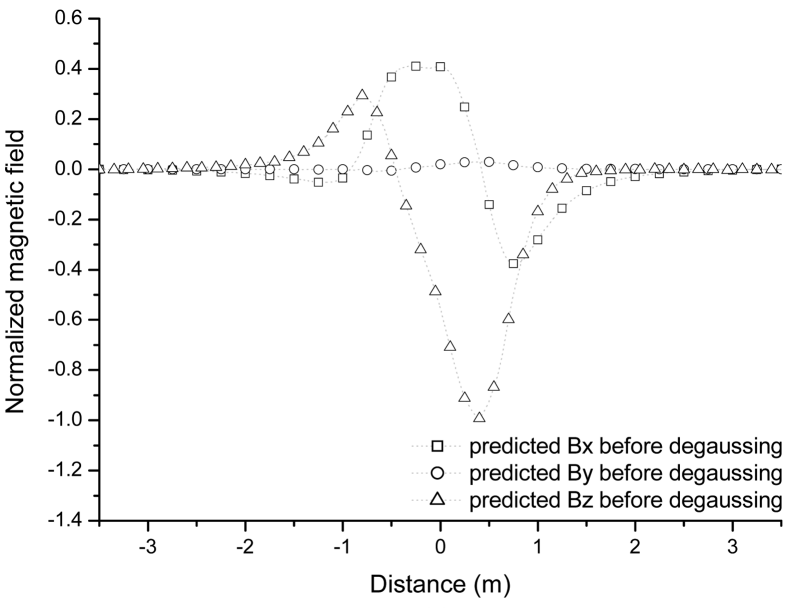

(a)

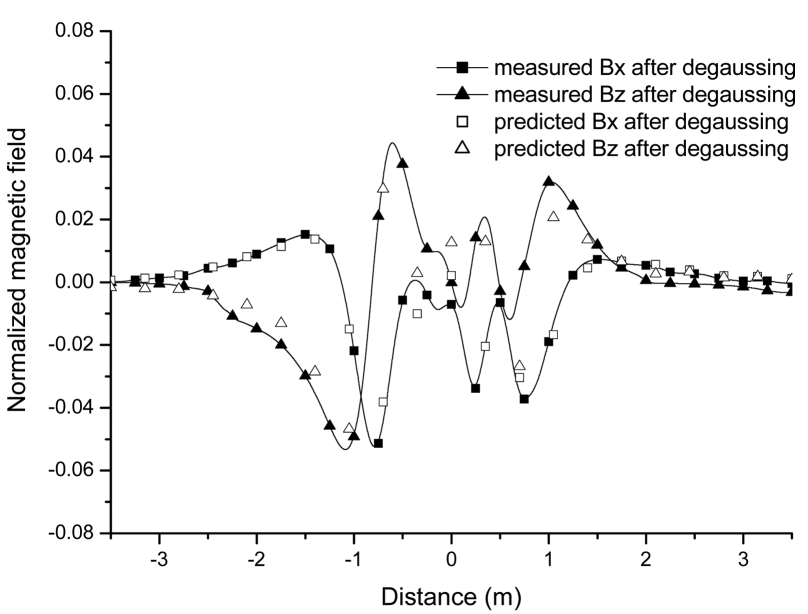

(b)

Fig. 5. Predicted coil field and magnetic fields after degaussing: (a) Coil field components, (b) predicted and measured field components after degaussing.

components of the composite coil field predicted after optimization. The composite coil field has very similar waveforms to IM field ones in Fig. 3, and their field directions are opposite with each other. The magnetic field predicted after degaussing is compared with measured one in Fig. 5(b). As seen in the figure, it is observed that the maximum field value is decreased by more than $95 \%$ compared to IM field before degaussing presented in Fig. 3.

\section{Proposed Re-degaussing Technique}

In practice, not IM and reference coil field data but optimum coil current values are recorded in a degaussing system equipped with a ship. Moreover, when a breakdown of onboard degaussing coils occurs at sea, MTF cannot be used for re-degaussing. In this section, a re-degaussing technique executable in such limited circumstances is 
explained. For a fast and efficient re-degaussing process, a new target field instead of IM field data is incorporated with an analytical $\mathrm{mmf}$ sensitivity formula, and then its numerical implementation is presented.

\subsection{Target field}

In order to estimate new optimum currents of available degaussing coils except for a faulty coil, a new target field corresponding to IM field should be defined. The target field can be easily constructed from the optimum current and arrangement information of degaussing coils before a coil breakdown. Each coil field is calculated from a simple integral equation describing the Biot-Savart law as feeding an allotted optimum current to a coil, one by one $[3,5,6]$. Thus, the target field is newly defined by the sum of approximated coil fields like Eq. (2).

$$
B_{i j}^{t a r}=\sum_{i=1}^{3} \sum_{j=1}^{n p} \sum_{k=1}^{n l} B_{i j}^{k}
$$

where $B_{i j}^{t a r}$ is three components of the target field, $B_{i j}^{k}$ is the $k$ th coil field components, the subscripts, $i$ and $j$, are the directional component and observation point, respectively, and $n l$ denotes the number of degaussing coils originally installed in a ship. It should be noticed that the target field does not include the shield effect of the hull, and also it may contain some numerical errors due to the restriction on realization of accurate coil arrangement.

Consequently, the aim of re-degaussing is to create a composite coil field as similar to Eq. (2) as possible by utilizing only available degaussing coils except for a faulty coil. This attempt is considered as the best possible way in an emergency situation where a part of degaussing coils is out of order.

\subsection{Analytical Sensitivity Formula}

For re-degaussing, an objective function $F$ is defined on the measuring line at a depth of $0.345 \mathrm{~m}$ under the keel as depicted in Fig. 4.

$$
\text { minimize } F=\sum_{i=1}^{3} \sum_{j=1}^{n p}\left(B_{i j}^{t a r}-\sum_{k=1}^{n m} B_{i j}^{k}\right)^{2}
$$

where $\mathrm{nm}$ denotes the number of available degaussing coils after a coil breakdown occurs. The second term $\sum_{k=1}^{n m} B_{i j}^{k}$ in Eq. (3) means a composite coil field constructed with only available degaussing coils except for a faulty coil. Herein, PM field is not taken into account because it does not change under both normal degaussing and coil breakdown conditions.

In order to search for an optimum of the inverse problem (3), an analytical mmf sensitivity formula is adopted [5-10]. In general, a sensitivity formula is derived by somewhat cumbersome mathematical procedures such as a governing Maxwell's equation, augmented objective function, and adjoint variable method. Detailed procedure of the sensitivity formula for magneto-static inverse problems has been well established from [5] to [8]. At this moment, only concern is to combine the sensitivity formula with the objective function (3).

Final mathematical expression of the sensitivity formula with respect to the coil $\mathrm{mmf}$ is given by Eq. (4).

$$
\frac{d F}{d \mathbf{p}}=\int_{\Omega}\left(\frac{\partial \mathfrak{I}}{\partial \mathbf{p}}\right) \cdot \lambda d \Omega
$$

where $\mathbf{p}$ is the system parameter (i.e. degaussing coil current), $\Omega$ is the cross-section of the coil, and $\lambda$ denotes the Lagrange multiplier interpreted as the adjoint vector potential. That is, it is a field solution of the adjoint system, which is the counterpart of a primary system [6]. At the iterative design during the optimization process, the pseudosource of the adjoint system is defined by differentiating Eq. (3) with respect to $B_{i j}^{k}$.

$$
\mathbf{M}_{j}=-2 \sum_{i=1}^{3}\left(B_{i j}^{t a r}-\sum_{k=1}^{n m} B_{i j}^{k}\right)
$$

where $\mathbf{M}_{j}$ corresponds to the pseudosource located at the $j$ th observation point, which can be interpreted as virtual magnetic dipole moment. It should be noticed that the degaussing coils have to be removed in the adjoint system. After all, the adjoint vector potential for the $l$ th coil is calculated by

$$
\lambda^{l}=\sum_{j=1}^{n p} \mathbf{M}_{j} \times \nabla\left(\frac{1}{r}\right)
$$

where $r$ is the distance from the pseudosource to the $l$ th coil position.

\subsection{Implementation}

To simplify numerical implementation, the coil mmf is forced to be a linear function of the system parameter $\mathbf{p}$ in (4) $[10,11]$. A general-purpose optimizer, called DOT based on the Broydon-Fletcher-Goldfarb-Shanno (BFGS) algorithm in [12], is adopted to accelerate the convergence of the objective function. The available degaussing coil currents are initially set to zero. The iterative design procedure for re-degaussing involves the following steps:

1) Prepare the target field data (2) with whole degaussing coils and define an objective function (3) with available coils except for a faulty coil.

2) Calculate a composite coil field in Eq. (3) for the available coils.

3) Assess the objective function (3) and then calculate the adjoint source (5). 
5) Compute the adjoint variable $\lambda$ in Eq. (6) and sensitivity value for individual coil with Eq. (4), respectively.

6) Update individual coil current value $I\left(I_{l}^{n k+1}=\right.$ $\left.I_{l}^{n k}+\alpha \Delta \mathbf{p}\right)$ at the $n k$ th iterative design, where $l$ and $\alpha$ is the $l$ th degaussing coil and relaxation factor, respectively.

7) Check convergence and go to step 2 if unsatisfactory.

\section{Case Study}

To examine the validity of the proposed re-degaussing technique, three faulty coil conditions are tested: $V_{2}$ coil breakdown, $L_{2}$ coil breakdown and $A_{2}$ coil breakdown in Fig. 1. This paper does not include the case that more than two degaussing coils are out of order because the range of coil power suppliers is limited and re-degaussing performance is abruptly degraded as well. The re-degaussing procedure is carried out with the coil arrangement information and coil current values optimized through the degaussing process. Before and after re-degaussing, magnetic fields of the model ship are recorded at a depth of $0.345 \mathrm{~m}$ under the keel line.

In Fig. 6, magnetic field distributions under a $V_{2}$ coil breakdown are compared with those in a normal degaussing condition before the breakdown. As expected, it is observed that the magnitude of $B_{z}$ is mainly increased by more than nine times after the coil breakdown occurs. To mitigate the increased field components, the proposed redegaussing procedure was executed for three available $V$ coils $\left(V_{1}, V_{3}\right.$ and $\left.V_{4}\right)$, and accordingly their current values were reassessed in a second. Fig. 7(a) shows current histograms where slash bars mean new coil currents optimized through re-degaussing and white bars denote the degaussing currents in the normal condition. From the result, it can be deduced that the increased currents of $V_{1}$

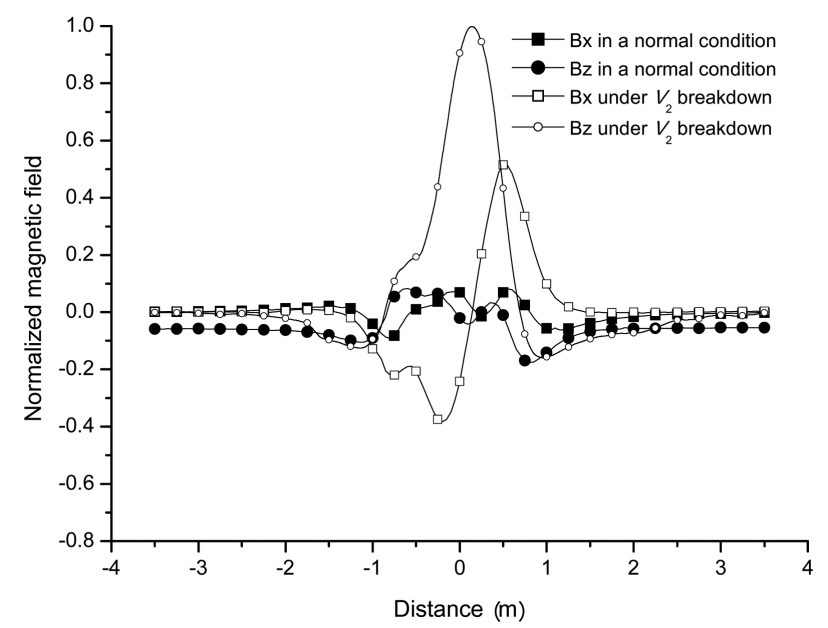

Fig. 6. Field components measured before and after $V_{2}$ coil breakdown.

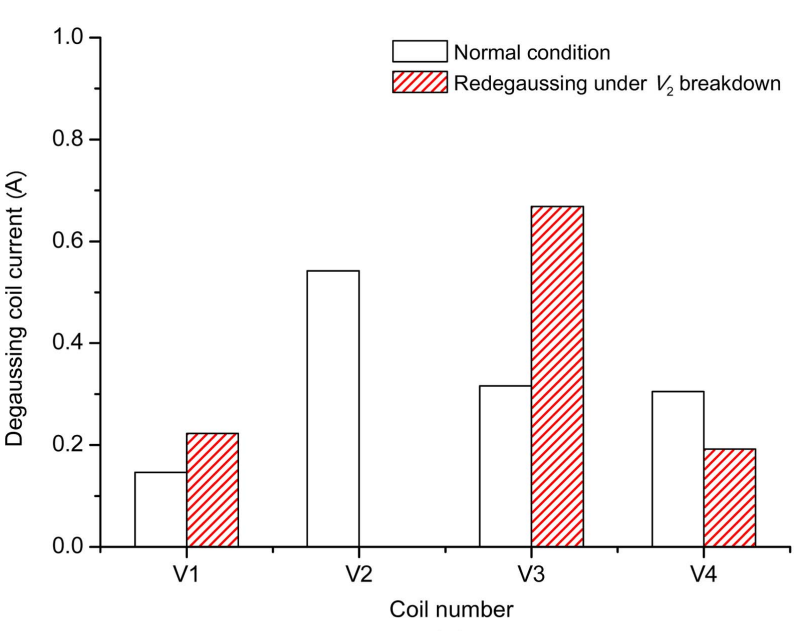

(a)

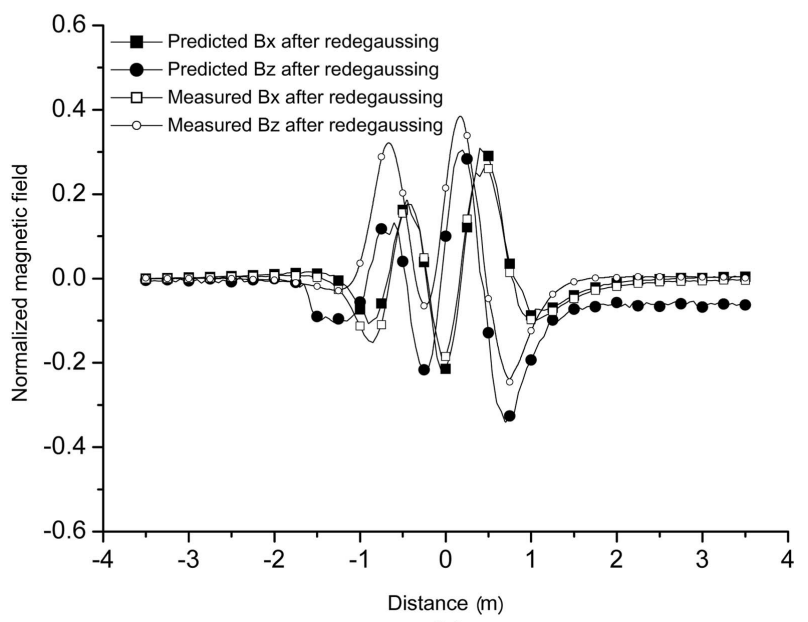

(b)

Fig. 7. (Color online) Comparison of $V$ coil currents and field components: (a) $V$ coil currents before and after re-degaussing, (b) predicted and measured field components after redegaussing.

and $V_{3}$ coils compensates for the loss of $V_{2}$ coil. The predicted field profiles after re-degaussing are compared with measured ones in Fig. 7(b), where some deviations appear in terms of field magnitudes and waveforms. It results from the fact that the proposed method basically uses not highly accurate numerical field solutions but rather the field integral equation approximated without considering the shielding effects of the hull. However, it is obvious that the measured field magnitude under the $V_{2}$ coil breakdown was reduced by more than $60 \%$ through the re-degaussing process.

The other two breakdown conditions of $L_{2}$ and $A_{2}$ coils are investigated from Fig. 8 to Fig. 11 as the same manner. In the case of a $L_{2}$ coil breakdown, both magnitudes of $\mathrm{B}_{\mathrm{x}}$ and $B_{z}$ are nearly four times as large as before the breakdown as seen in Fig. 8. Except for $L_{2}$ coil, optimum currents of six available $L$ coils were newly calculated as 


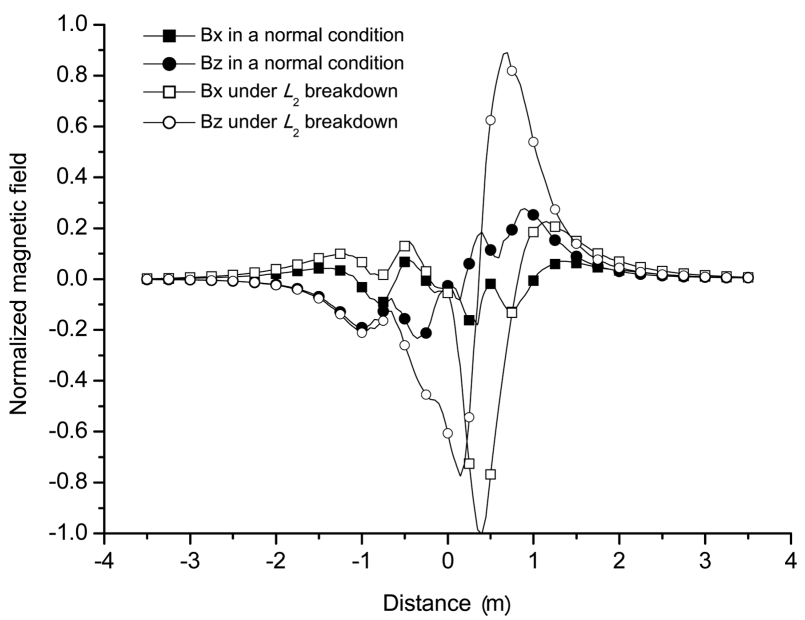

Fig. 8. Field components measured before and after $L_{2}$ coil breakdown.

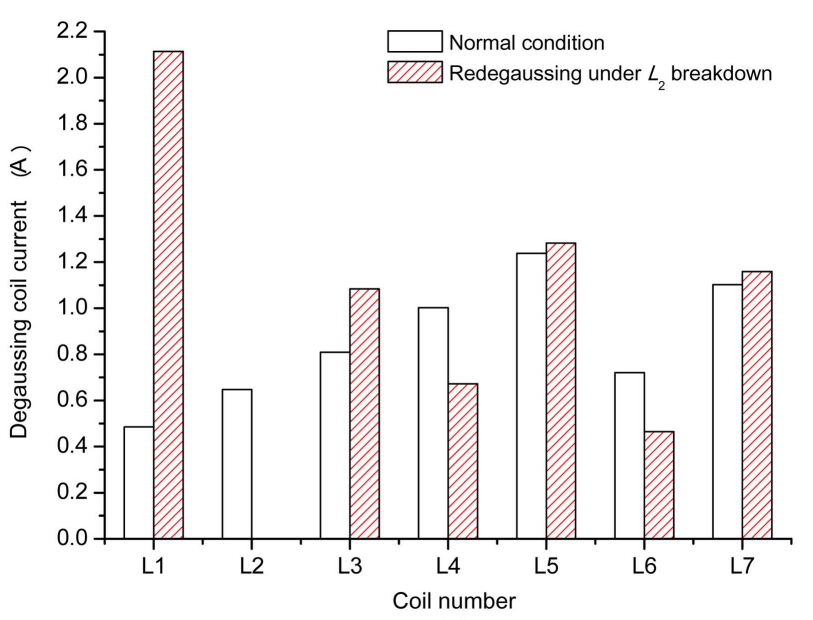

(a)

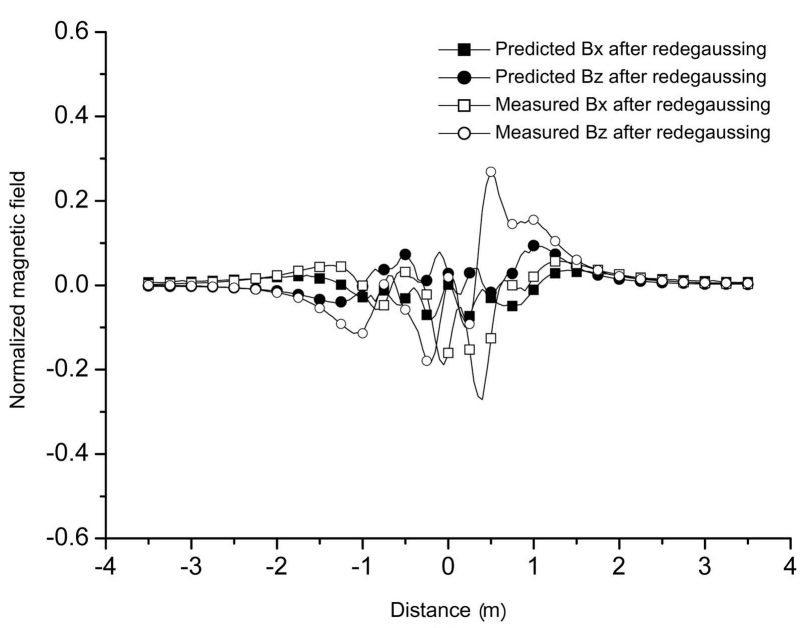

(b)

Fig. 9. (Color online) Comparison of $L$ coil currents and field components: (a) $L$ coil currents before and after re-degaussing, (b) predicted and measured field components after redegaussing.

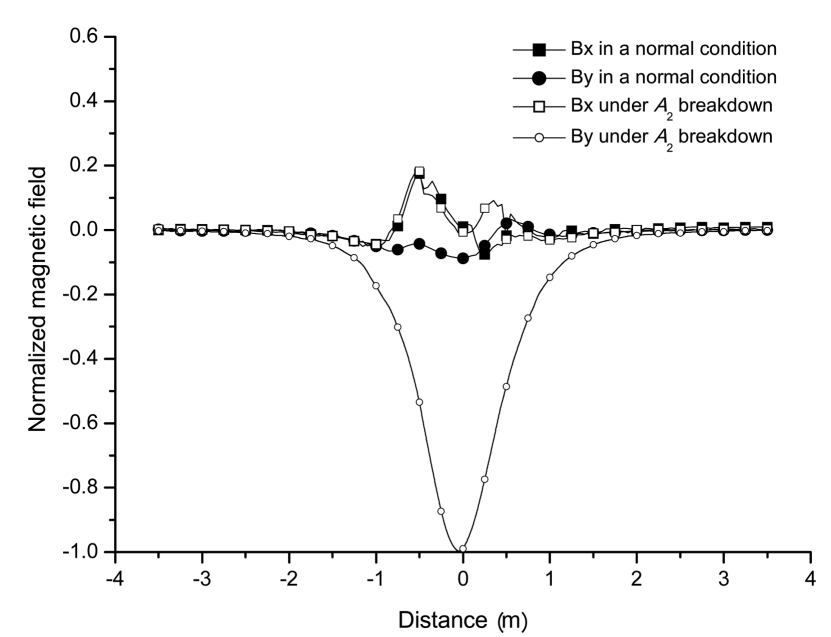

Fig. 10. Field components measured before and after $A_{2}$ coil breakdown.

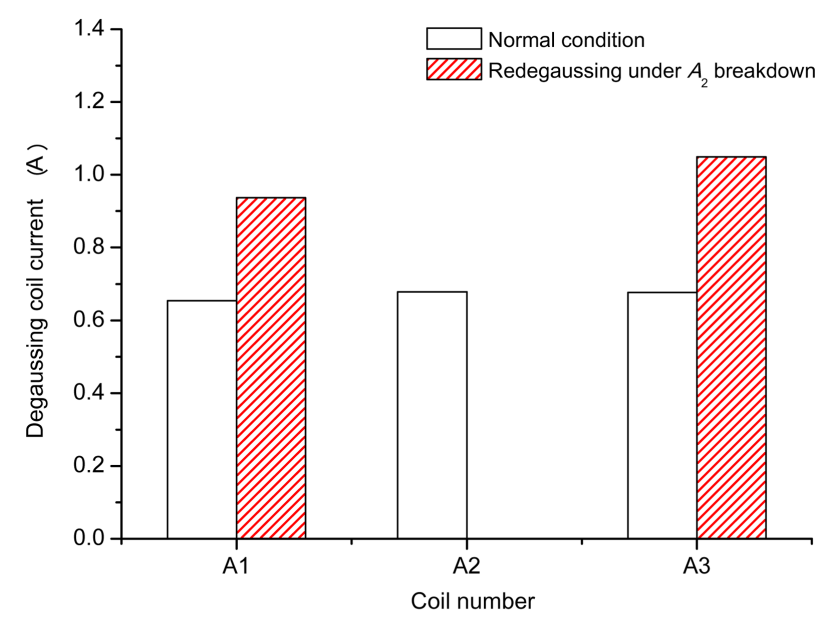

(a)

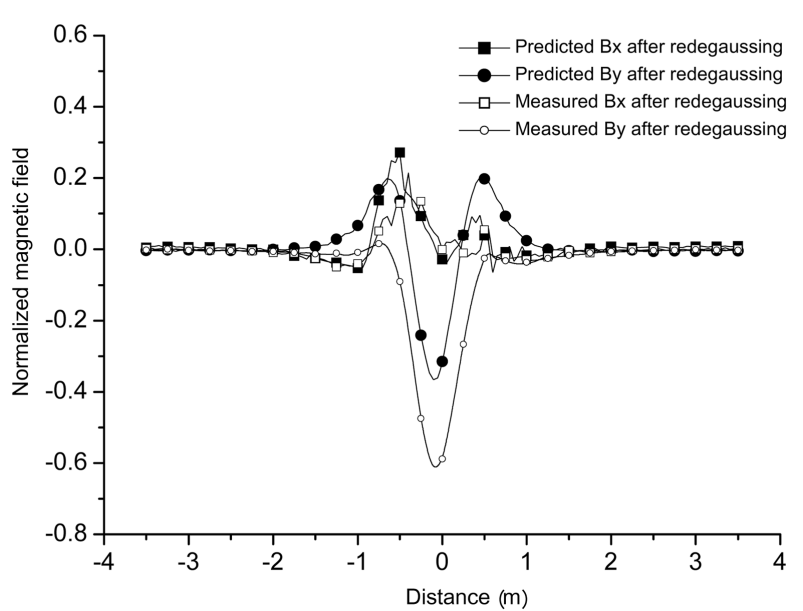

(b)

Fig. 11. (Color online) Comparison of $A$ coil currents and field components: (a) $A$ coil currents before and after redegaussing, (b) predicted and measured field components after re-degaussing. 
seen in Fig. 9(a). It is observed that the $L_{1}$ coil current after re-degaussing are more than three times larger than that in the normal degaussing condition. It comes from the reason that the $L_{1}$ coil size is much smaller than the others in Fig. 1(a), and accordingly a relatively high current is allotted to compensate for the loss of $L_{2}$ coil. Fig. 9(b) shows that the measured field magnitude under the $L_{2}$ coil breakdown was reduced by more than $70 \%$ through the proposed re-degaussing process.

When a $A_{2}$ coil breakdown occurs, the magnitude of $\mathrm{B}_{\mathrm{y}}$ in Fig. 10 are mainly increased by more than ten times that in the normal degaussing condition. In this case, only two coils $\left(A_{1}\right.$ and $\left.A_{3}\right)$ are available for re-degaussing because three $A$ coils were originally installed in the scaled ship shown in Fig. 1. The current histograms after re-degaussing are compared with those in the normal condition in Fig. 11(a), where $A_{1}$ and $A_{3}$ currents are increased to compensate for the loss of $A_{2}$ coil. In Fig. 11(b), it is observed that the measured field magnitude under the $A_{2}$ coil breakdown was reduced by more than $40 \%$ through the re-degaussing process.

Form the results, it can be inferred that the more available coils, when a degaussing coil breakdown occurs, the bigger the contribution to improving re-degaussing performance.

\section{Conclusion}

In this paper, an efficient and practical re-degaussing technique for a ship experiencing a breakdown of onboard degaussing coils at sea is proposed and tested with a model ship equipped with 14 degaussing coils. Utilizing an analytical mmf sensitivity formula and approximated coil field, new optimum currents of available coils are successfully obtained under the limited information of normal degaussing coil currents and coil arrangement. Through three faulty coil conditions conducted in a scale- down MTF test room, it is verified that the proposed method yields fast and efficient re-degaussing results.

\section{Acknowledgment}

This work has been supported by the Low Observable Technology Research Center program of Defense Acquisition Program Administration and Agency for Defense Development.

\section{References}

[1] R. Donati and J. P. Le Cadre, IEE Proc. Radar Sonar Navig. 149, 221 (2002).

[2] O. Chadebec, J. Coulomb, J. Bongiraud, G. Cauffet, and P. Thiec, IEEE Trans. Magn. 38, 1005 (2002).

[3] O. Chadebec, J. Coulomb, G. Cauffet, and J. Bongiraud, IEEE Trans. Magn. 39, 1634 (2003).

[4] H. Liu and Z. Ma, Proc. Int. Conf. Mechatronics and Automation 3133 (2007).

[5] C. Yang, K. Lee, G. Jung. H. Chung, J. Park, and D. Kim, J. Appl. Physics 103, 905 (2008).

[6] J. Lee, H. Choi, W. Nah, I. Park, J. Kang, J. Joo, J. Byun, Y. Kwon, M. Sohn and S. Kim, IEEE Trans. Appl. Supercond. 14, 1906 (2004).

[7] M. Minakami, IEEE Trans. Appl. Supercond. 14, 940 (2004).

[8] N. Choi, G. Jeung, C. Yang, H. Chung, and D. Kim, IEEE Trans. Appl. Supercond. 42, 4904504 (2012).

[9] N. Choi, G. Jeung, S. Jung, C. Yang, H. Chung, and D. Kim, IEEE Trans. Magn. 48, 419 (2012).

[10] K. Lee, H. Choi, W. Nah, I. Park, J. Kang, J. Joo, J. Byun, Y. Kwon, M. Sohn, and S. Kim, IEEE Trans. Magn. 45, 1478 (2009).

[11] D. Kim, J. Sykulski, and D. Lowther, IEEE Trans. Magn. 41, 1752 (2005).

[12] DOT User Manual, Vanderplaats Research \& Development Inc., Colorado Springs, USA (2001). 